\section{On the Sub-Nanometer Resolution of Scanning Electron and Helium Ion Microscopes}

András E. Vladár, Michael T. Postek, and Bin Ming National Institute of Standards and Technology (NIST) ${ }^{1}$

Gaithersburg, MD 20899-8212

andras@nist.gov

\section{Introduction}

All forms of microscopy are being pushed to the limit by nanotechnology. $[1,2]^{12}$ This is especially true for high-resolution scanning electron and helium ion microscopes, which are proving to be extremely useful for nanometer-scale imaging, characterization, and dimensional measurements. Excellent resolution is essential for imaging nanomaterials. Hence, there is a relentless quest to achieve better and better resolution with various electron and ion microscopes and to monitor and maintain these instruments to achieve the best possible performance levels.

The ability to resolve fine details with a microscope has greatly improved over the past 20 years. The resolution achievable with the newest scanning electron microscopes (SEMs) can now be at or below $0.4 \mathrm{~nm} \mathrm{[3]}{ }^{3}$ and for the scanning helium ion microscope (HeIM), $0.24 \mathrm{~nm}$ has been reported [4]. ${ }^{4}$ To put this into perspective, the $\{111\}$ crystal plane in silicon $(\mathrm{Si})$ has $0.32 \mathrm{~nm}$ lattice spacing, so one cubic nanometer in the Si crystal contains only a few atoms. Therefore, an instrument must be performing at the highest level possible to resolve routinely the finest structures. Clearly, the instrument operator must also be functioning at a high level as well to achieve the best resolution and to prove that the instrument is capable of doing the work.

Determining the resolution achievable with a scanning electron or ion microscope is an elusive task. There are several definitions of resolution and a number of ways of calculating it. Only some of the methods are repeatable and none of them is accurate or traceable. With SEMs and HeIMs today it is impossible to resolve atoms in crystals like it is feasible in some transmission electron microscopes (TEMs), which would offer accurate resolution measurements. Still, the trained human eye is able to differentiate good images from inferior ones depending on their sharpness. [5] $\mathrm{T}^{5}$ he capability of doing so in objective and repeatable ways will be discussed later in this paper.

A number of factors contribute to attainable resolution of an instrument such as the physics of the particle beam and the focusing ability of the electron or ion-optical column, the size and shape of the best focus, the conical angle of the beam, and the electron- or ion-distribution within the beam. The resolution also depends on the sample since the size and shape of the information volume, i.e. that region of the excited volume where the collected (generally) secondary electron signal originates. Moreover, the amount of noise present in the system plays an important role as well, because it limits the amount of informa-

1. Contribution of the National Institute of Standards and Technology; not subject to copyright. Certain commercial equipment is identified in this report to adequately describe the experimental procedure. Such identification does not imply recommendation or endorsement by the National Institute of Standards and Technology, nor does it imply that the equipment identified is necessarily the best available for the purpose. tion recoverable from the image generated by the microscope. In almost all practical cases, the focusing ability of the optical column is not the limit of the resolution, because other factors, including environmental effects, electro-magnetic fields, sample stage and beam drift, and vibration are also at work and may reduce the achievable resolution.

\section{The Primary Electron and Ion Beams and Excited Volumes}

The primary electron or ion beam is composed of a threedimensional distribution of electrons or ions along and across the beam. It is generally thought to have a somewhat asymmetric double-conical shape, with the important half-angle parameter that defines the depth-of-field, and the disk of least confusion, where the best focus, i.e. the smallest spot size can be achieved. Figure 1 shows an idealized representation of the electron or ion beam on the left. In this case there is a $3 \mathrm{~nm}$ difference in the beam diameter at the top and at the bottom of the $100 \mathrm{~nm}$ trapezoid structure.

Narrower beams result in smaller differences. They also result in better depth-of-field. Figure 1 on the right shows the region (depicted as a small cylinder) where the sample can be considered in focus and its image sharp. This region, i.e. the depth-of-field diminishes with growing half angle and beam spot size.
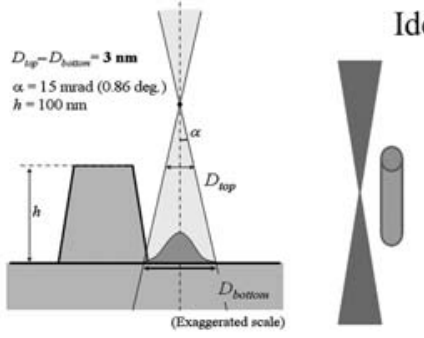

Ideal cases

Practical cases

Figure 1. Idealized electron or ion beam with double-conical shape and Gaussian electron or ion intensity distribution across the beam (left, courtesy of Maki Tanaka of Hitachi High Technologies) and the sharp region, i.e. depth-of-field, depicted by cylinders that diminish with increasing half angle and beam spot size (right).

Depending on the parameters used, the HeIM can produce a much narrower beam than the SEM because the ion source is a single atom. $[6,7]{ }^{67}$ Therefore, the depth-of-field can be larger than the SEM even at high magnifications, thus resulting in a sharper image overall. The comparison of SEM imaging to HeIM imaging of the same sample of gold $(\mathrm{Au})$ coated tin balls shown in Figure 2 illustrates this point. Note that the top of the large tin balls in the SEM image (left) is quite sharp, but smaller structures in the background are not as sharp to the eye. Contrast that with the HeIM image (right) where the tin balls are sharp in both the foreground and the background. ${ }^{2}$ Even though both of the images were taken by collecting secondary electrons, they look different because of the differences between the two instruments, excitation beams, signal generation, and collection.

The signal from the SEM or the HeIM is a complex product of: 1) the interaction of the electron or ion probe, 2) the sample, 3) sample chamber geometry and chamber material, 4) and the electro-magnetic field present around the sample (either from the instrument itself or from sample biasing and charging). The

2. It must be noted that the ability to identify subtle differences in the micrographs will be a function of the printing process. The original images are available from the authors. 


\section{ORIONं PLUS \\ World Record Resolution \\ Changes Microscopy Paradigm}
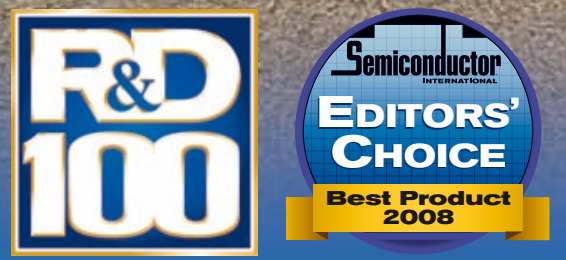

\section{ZHISS ORION'PLUS.}

The Award winning helium ion microscope for revolutionary cutting edge imaging applications, providing ultra high resolution coupled with unique material contrast.

World Record!

Image resolution of $0.24 \mathrm{~nm}$ in SE mode
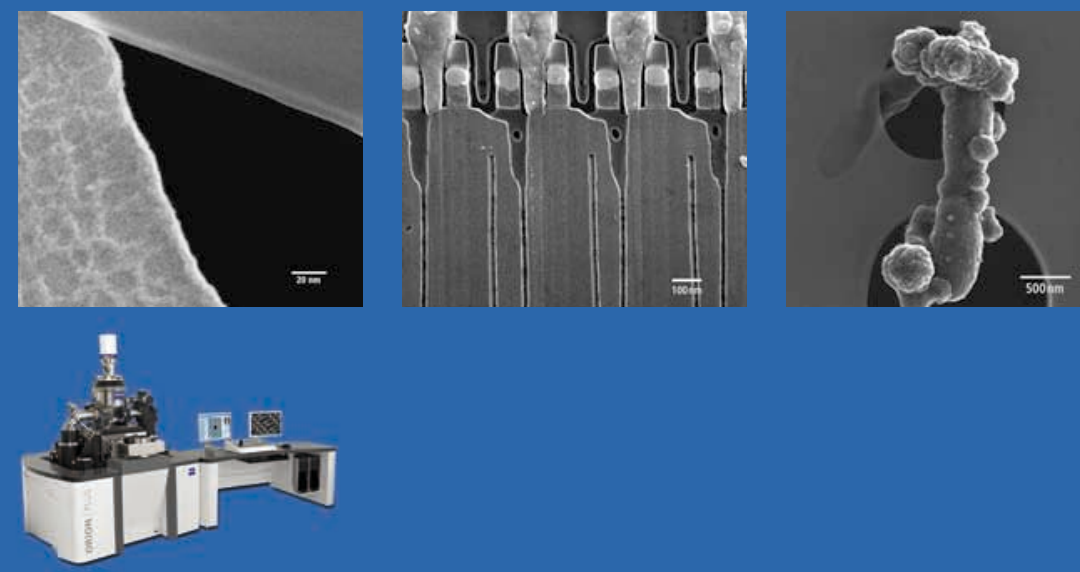

ORION ${ }^{\circledR}$ PLUS - Imagine seeing things for the very first time

Carl Zeiss SMT Inc.
One Corporation Way
Tel. $+1978 / 8267909$ Fax $+1978 / 5325696$ info-usa@smt.zeiss.com www.zeiss.com/nts 


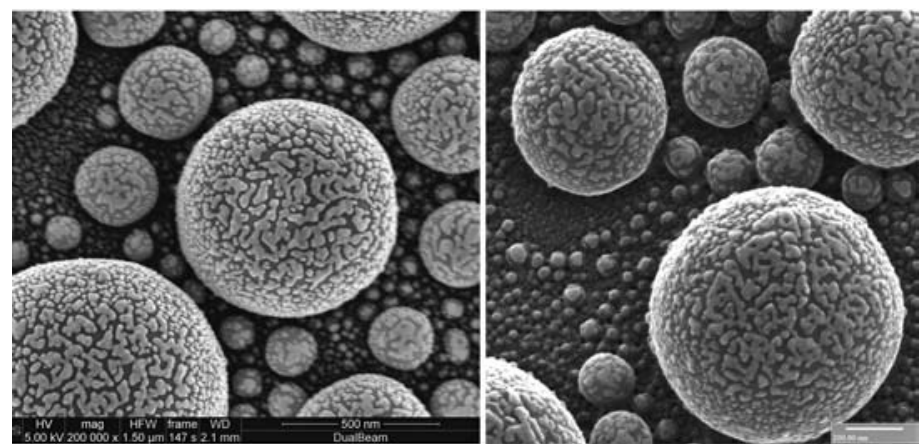

Figure $2.5 \mathrm{keV}$ landing energy SEM image of an Au-decorated tin ball sample (left). The same sample imaged in high vacuum with $31 \mathrm{keV}$ landing energy HeIM (right). The field of view for both micrographs is $1.5 \mu \mathrm{m}$ ).

three-dimensional electron or ion beam and the sample define the $3 \mathrm{D}$ information volume, which is that part of the $3 \mathrm{D}$ excited volume where the detected signals come from. Therefore, although imaging in the SEM or HeIM seems straightforward, this is in reality a rather complex affair. Additionally, for accurate dimensional measurements at the nanometer scale, sophisticated modeling methods that account for all the physical processes must be used to extract the shape and size of the sample structures of interest.

The differences in excited volumes of the SEM and the HeIM are illustrated in Figure 3. This figure schematically illustrates the current understanding of the nature of the excited volumes of the SEM (left) and the HeIM (right). This volume is where the important beam-specimen interactions take place. The SE escape depth is the region from whence the secondary electrons have enough energy to leave the sample surface. The escape depth can be a few nanometers to up to $10 \mathrm{~nm}$, depending on the sample material. For metals it is thinner, for insulators it is generally larger.

The size and shape of the excited and information volumes along with the secondary electron (SE) generation efficiency and location are important for another reason. The amount of surfacerelated information collected is directly dependent on these factors. Those secondary electrons that carry information about the finest details of the sample are generated by the primary electrons or ions at the point where the beam hits the sample. These are the

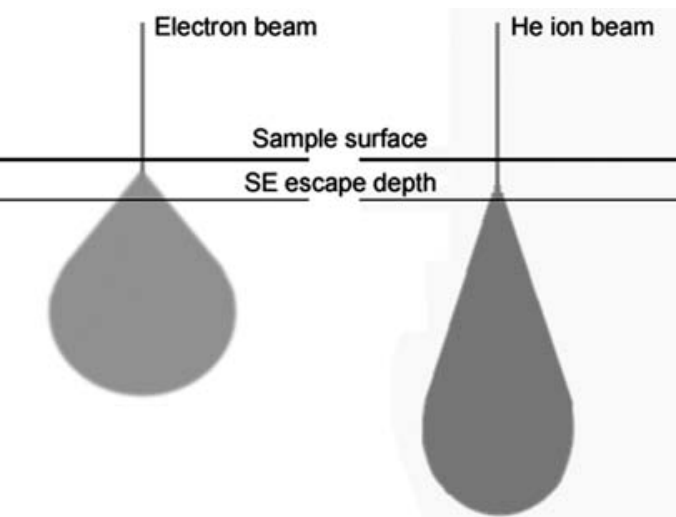

Figure 3. Schematic illustration of the excited volumes of the SEM (left) and the HeIM (right), where the beam-specimen interactions take place. The SE escape depth is the region from whence the secondary electrons have enough energy to leave the sample surface. emerge from, SE2 electrons do not carry information about the finest sample details; in fact, it is thought that they can emerge from a much larger area around the point of impact of the primary beam. The size of this area depends on the primary excitation energy, the shape, and the material composition of the sample and could reach over more than a micrometer. Electrons are also generated by the backscattered electrons or ions that leave the sample and hit some other material within the sample chamber. These secondary electrons are called SE3. Again, these do not carry information about finest sample details. The well-focused beam always generates SE1 electrons, but the amount and the ratio of SE1 and SE2 and SE3 electrons have a profound effect on the appearance and the amount of fine details of the secondary electron image. This is especially true in the case of the HeIM. In the HeIM, secondary electrons are produced at (or very nearwithin the escape depth of the SEs) the point of initial interaction with the sample and thus, are equivalent to SE1 electrons of the SEM. These initial SEs are generated in large numbers and produce images with strong and familiar topographic contrast, which are very similar to the secondary electron images obtained from an SEM. But, in the HeIM, in many instances, the surface detail is enhanced because, contrary to the SEM interactions it is believed that the ion beam passes much more deeply into the sample matrix and few SE2 or SE3 type electrons characteristic of the SEM are generated. The flood of SE2 and SE3 electrons resulting from the backscatter of electrons in the SEM can to some extent "wash-out" surface detail potentially resolved by the electron beam. This does not occur in the HeIM resulting in enhanced surface detail. Figure 4. shows an example for the rich surface details provided by the HeIM, even at high landing energies.

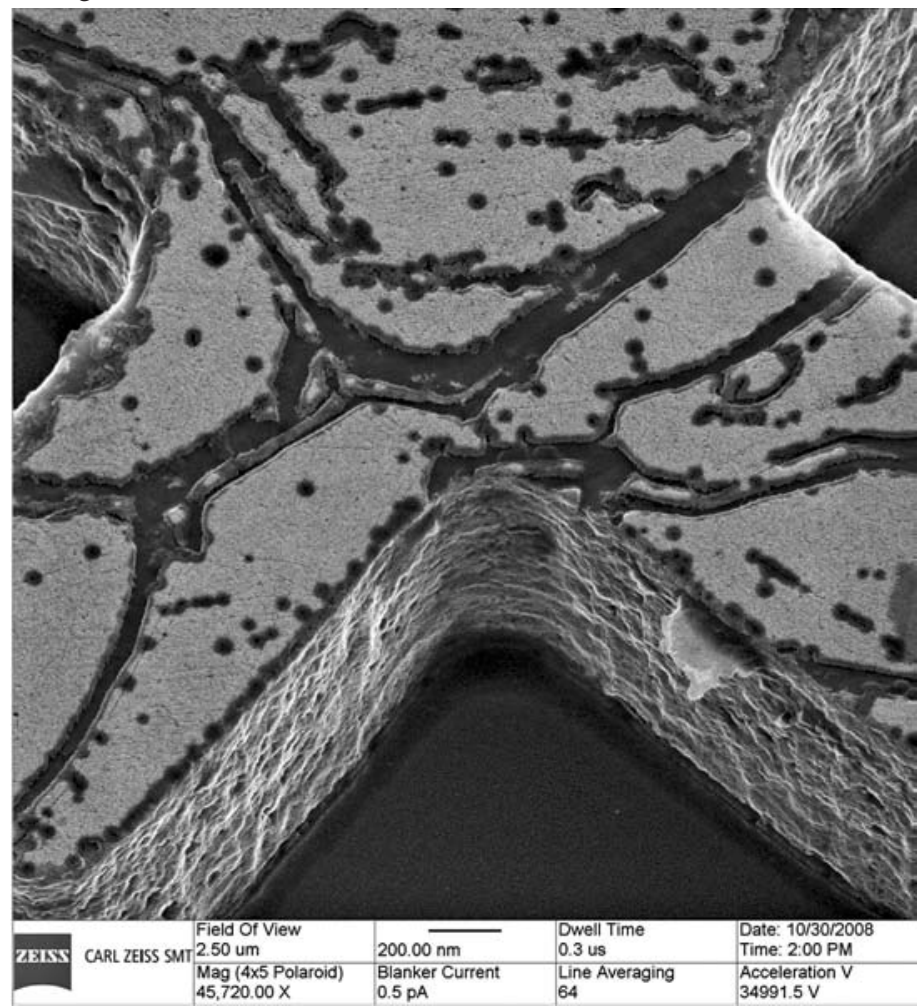

Figure $4.35 \mathrm{keV}$ landing energy HeIM SE image of a contaminated Al marker showing excellent surface details and depth of field. The field of view is $2.5 \mu \mathrm{m}$ (Courtesy of Carl Zeiss SMT).

so-called SE1 electrons. The s e c ond ary electrons that are created by energetic electrons or ions backscattered within the sample and leave the sample are called SE2 electrons.

Because of the size and location of the region they 


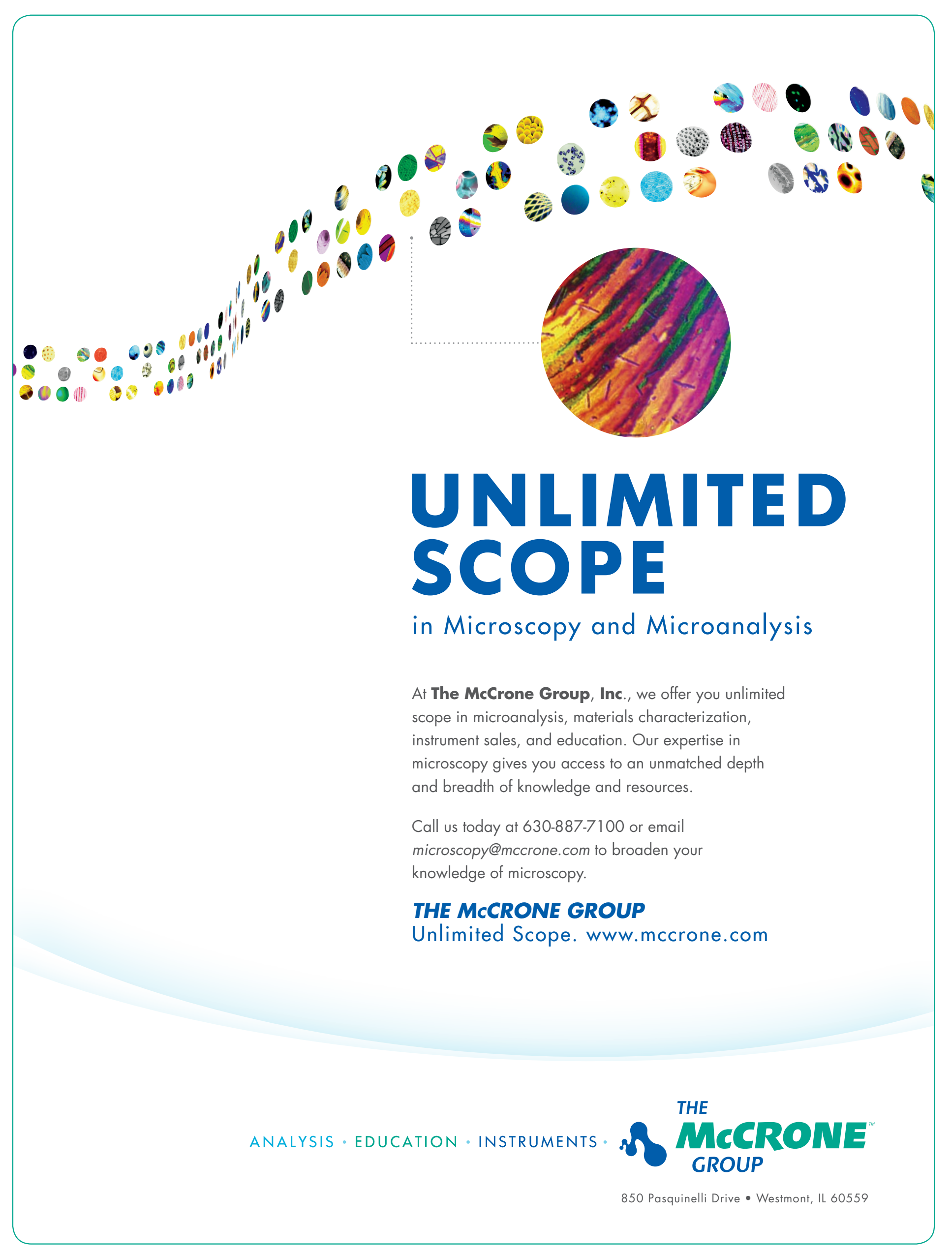


Resolution Measurements for the Scanning Electron and Ion Microscopes

SEMs, historically, have been used primarily for generating images, and for x-ray microanalysis. In recent years, specialized instruments have been developed and are used routinely for high-speed dimensional measurements in the production lines of integrated circuit manufacturers. HeIMs are relatively new and mostly used for imaging. Other analytical methods are still in development.

As stated above, one of the most important SEM or HeIM parameters is the spatial resolution of the image. All these instruments are characterized by some kind of best attainable, guaranteed resolution. Resolution is one of the key parameters for instrument acceptance at and after installation and for tracking the daily performance of the instrument. Yet, it is one of most elusive and least understood parameters. The measured "spatial resolution of the SEM (or HeIM)" is a highly simplified numerical representation of the complex imaging system and imaging process of these instruments. Today, manufacturers use various methods to "prove" the resolution of the instruments. One common approach is the measurement of the gap between two very fine particles or structures. Figure 5 shows an example of the gap-based resolution measurement. Usually evaporated gold-on-carbon, platinum-palladium-carbon or gold-evaporated magnetic tape samples are used for this measurement. Once the magnification or scale is correctly calibrated, an image is taken at a suitably high magnification and finally the width of the smallest gap is found, measured, and presented as the best resolution of that instrument.

Alternative methods include the calculation of transition of the video signal using pre-set values (e.g. $25 \%$ to $75 \%$ ). Figure 6 shows the scanning helium ion microscope image of an asbestos fiber on a thin holey carbon foil sample. This signal transitionbased resolution measurement yielded $0.24 \mathrm{~nm}$ resolution. In the past, other software algorithms were developed to lessen the effects of the human factor in the measurement. Such algorithms

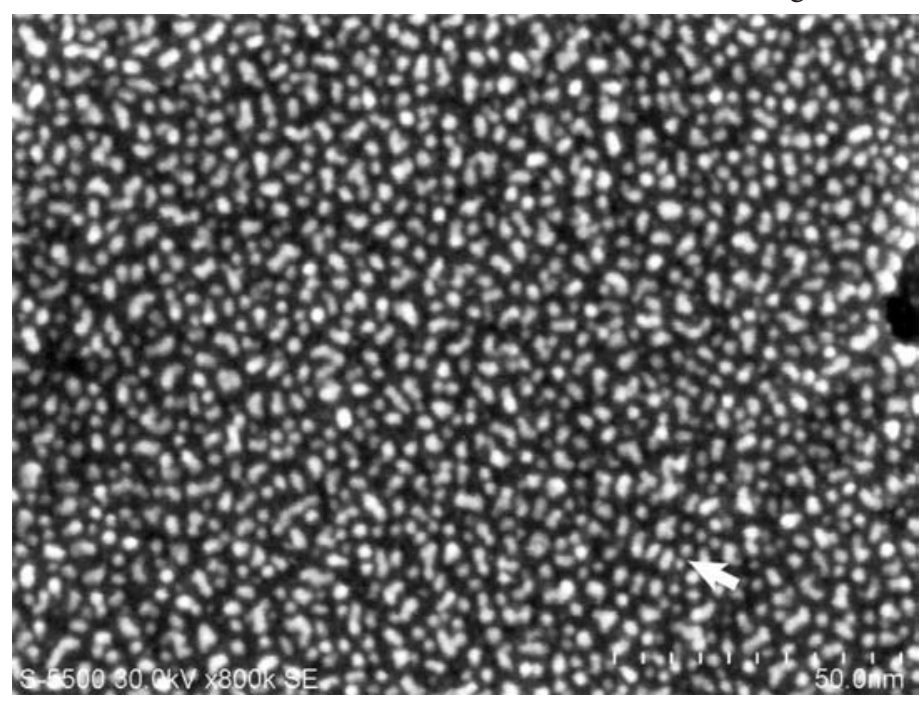

Figure 5. Example of a gap-based resolution measurement on Pt-Pdon-carbon sample. The arrow points to a $0.4 \mathrm{~nm}$ gap on an image taken on an in-lens high resolution field emission SEM. The image shown proves that its resolution specification was met. The field-of-view is $160 \mathrm{~nm}$ (Courtesy of Hitachi High Technologies). were initially published by Postek and Vladar [5], Vladar et al. [7], Zhang et al. [8], and by Joy [10].

\section{Image Sharpness International Standard Development}

High instrument resolution is the "holy grail" of scanning electron and ion microscopy. The goal is to be able to see and measure the smallest details on the samples. Table 1 shows the manufacturer specified resolution values of four contemporary scanning electron and ion microscopes installed within the Precision Engineering Division of NIST. The methods used in obtaining these specification numbers are diverse (as described above) and none of them are "accurate" or traceable to internationally

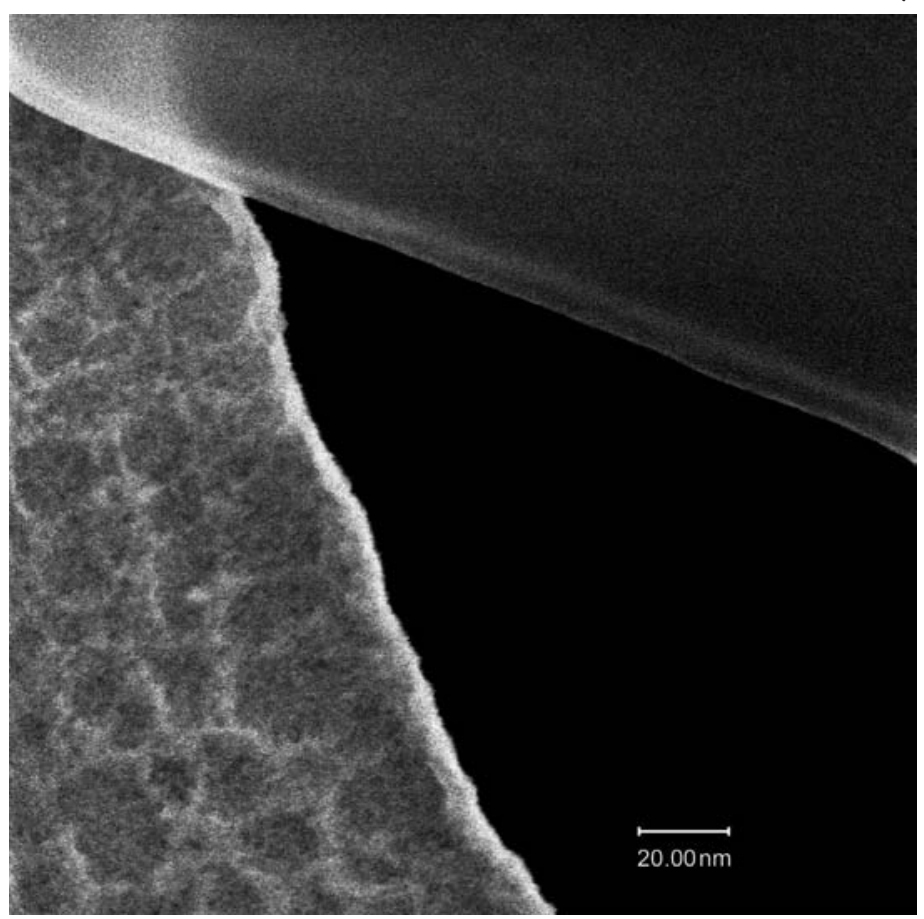

Figure 6. Example of a signal transition-based resolution measurement on an asbestos fiber on a thin holey carbon foil sample. The quoted resolution is $0.24 \mathrm{~nm}$ on an image taken on an Orion Plus HeIM. The field-of-view is $200 \mathrm{~nm}$ (Courtesy of Carl Zeiss SMT).

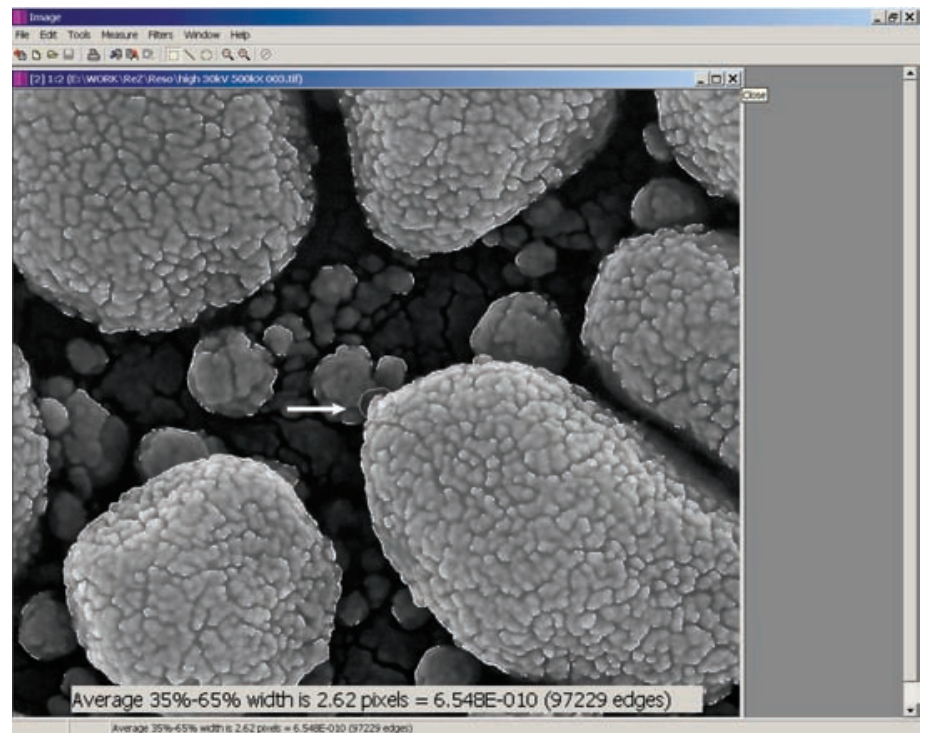

Figure 7. Computerized calculation-based resolution measurement method showing $0.65 \mathrm{~nm}$ spatial resolution performance of the SEM. Note the circular beam shape in the center of the image. The field-of-view of the Pt decorated gold-on carbon sample is $512 \mathrm{~nm}$. 


\section{Let 4pi take you beyond the capability of any SEM/STEM on the market with}

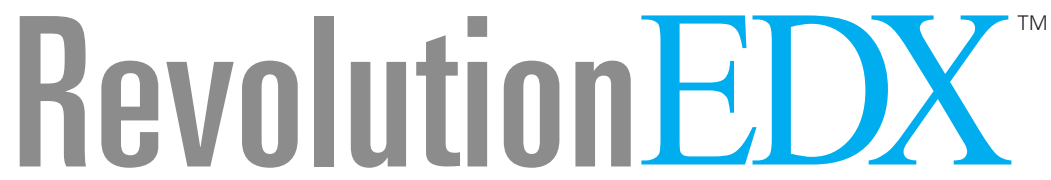

X-ray Microanalysis Systems

Save time and production costs with 4pi's unique features

Features available exclusively from $4 \mathrm{pi}$ :

Event-Streamed Spectrum Imaging ${ }^{\text {m' }}$

and

Spatial Frame Lock ${ }^{\text {TM }}$

Real-time electron-beam drift correction
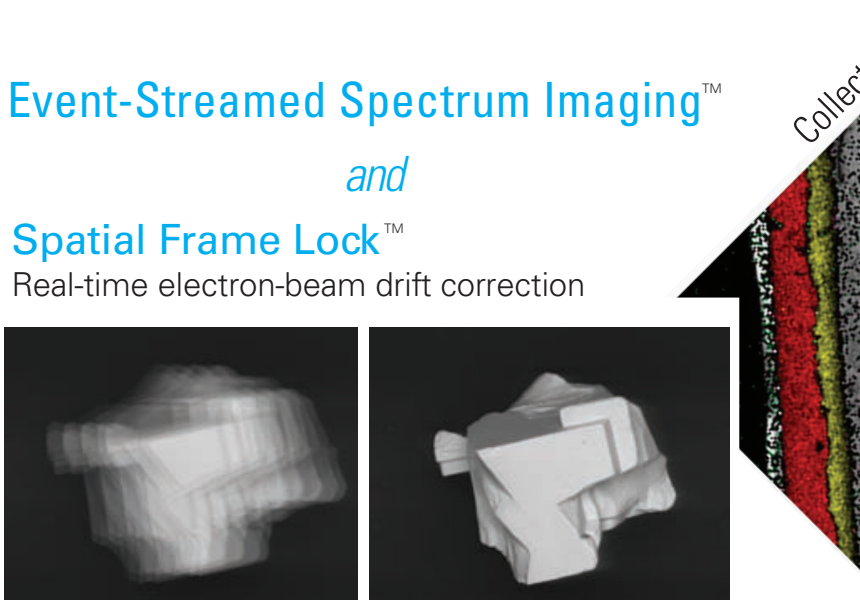
accepted standards today. Clearly, this makes direct comparisons very complicated. Standardization of instrument performance is one of the goals of the NIST Manufacturing Engineering Laboratory's Nanomanufacturing and Next-Generation Metrology Programs; for which understanding the overall behavior of the individual instruments is indispensable. The four instruments measured all reside in environmentally stable laboratories within the NIST Advanced Measurement Laboratory. All of the manufacturers have generously collaborated with NIST to maintain these instruments at the highest possible levels of performance.

Resolution of Scanning Electron and Ion Microscopes

\begin{tabular}{|c|c|c|c|}
\hline & $\begin{array}{c}\text { Manufacturer } \\
\text { Specification } \\
(\mathrm{nm})\end{array}$ & Best Measured (nm) & $\begin{array}{c}\text { Median of } \\
\text { Measured }\end{array}$ \\
\hline Instrument 1 & 0.9 & 0.65 & 0.78 \\
\hline Instrument 2 & 0.4 & 0.38 & 0.42 \\
\hline Instrument 3 & 0.8 & 0.7 & 1.2 \\
\hline Instrument 4 & 1.0 & 0.8 & 0.9 \\
\hline
\end{tabular}

** Instrument identification and manufacturer intentionally removed.

Table 1. The resolution measurement results obtained on four different instruments located in NIST. The data reported in the table were obtained by the use of proprietary software, which is currently under evaluation at NIST. The data for the median measured were obtained over many weeks.

Clearly, Table 1 represents a snapshot in time and as technology improves, so will the instruments' resolution. Instrument resolution is constantly being improved upon by instrument manufacturers. Therefore, resolution has been and will remain a fiercely competed specification by the instrument manufacturers. As stated above, using the conventional method for evaluating the image resolution, the gap distance of the neighbouring particles in the image is measured by the human eyes based on the sharpness at the edges of these particles. This may result in error due to the human interaction in the procedure. As stated above, more objective image analysis methodologies have been published and have been applied in order to remove human error from the procedure $[5,8,9,10]$ and manufacturers have begun developing computer-based performance-monitoring embedded within their instruments. All of these must be fully tested to ensure that they are highly robust and repeatable and the analysis is appropriately and fairly undertaken. Recently, the development of a new standard for the evaluation of image sharpness has begun at the International Organization for Standardization (ISO) [11]10. Currently, a working committee of ISO is reviewing a draft documentary standard of software programs for the evaluation of image resolution in the SEM and HeIM.

Table 2 presents the results obtained with various software programs, including the ISO candidate algorithms, on some images obtained by various SEM and HeIM instruments. This evaluation is based on the full-size versions of the images in Figure 8 , and it is very limited in its scope. The sharpness values are reported in pixels, which were converted into a resolution-related number in nanometers using appropriate pixel/nanometer values.

\begin{tabular}{|c|c|c|c|c|c|c|c|c|}
\hline \multicolumn{10}{|c|}{ Methods } \\
\hline Image & 1 & 1 & 2 & 2 & 3 & 3 & 4 & 4 \\
\hline & pixels & $\mathrm{nm}$ & pixels & $\mathrm{nm}$ & pixels & $\mathrm{nm}$ & pixels & $\mathrm{nm}$ \\
\hline 1 & 2.42 & 1.35 & 4.72 & 2.63 & 5.62 & 3.13 & 3.48 & 1.94 \\
\hline 2 & 5.37 & 0.39 & 10.74 & 0.77 & 12.20 & 0.88 & 16.03 & 1.15 \\
\hline 3 & 6.67 & 0.65 & 11.47 & 1.12 & 39.40 & 3.86 & 12.38 & 1.21 \\
\hline 4 & 3.77 & 0.47 & 8.66 & 1.08 & 13.00 & 1.61 & 8.42 & 1.05 \\
\hline 5 & 2.36 & 0.65 & 4.63 & 1.16 & 7.10 & 1.78 & 5.04 & 1.26 \\
\hline 6 & 3.71 & 0.36 & 8.07 & 0.79 & 12.30 & 1.20 & 9.35 & 0.91 \\
\hline 7 & 4.31 & 0.42 & 8.07 & 0.79 & 14.9 & 1.45 & 6.14 & 0.60 \\
\hline 8 & 2.64 & 0.53 & 5.18 & 1.04 & 7.29 & 1.46 & 4.34 & 0.87 \\
\hline 9 & 1.51 & 2.2 & 3.14 & 4.58 & 3.43 & 5.01 & 2.38 & 3.47 \\
\hline
\end{tabular}

Table 2. The results of four image sharpness and resolution calculation algorithms.

It is clear from these data that the numbers computed using each method are different, as there are no universal, accurate methods for sharpness and resolution measurement yet. As our knowledge improves, it will be possible to get these results closer to each other. The correlation coefficients among the various methods are: Method 1 to Method 2 is 0.97 , to Method 3 is 0.88 and to Method 4 is 0.87 . All show high levels of correlation among the results obtained on images of a very diverse set of samples, taken at various magnifications and on different SEM and HeIM instruments.

Despite the limited nature of this evaluation and the obvious numerical differences, each software program gives a credible performance judgment on the relative sharpness characteristics among the nine images. The results indicate that successful tracking of image sharpness is indeed possible. Further improvements and the adoption of consistent sample types and measurement conditions will improve the correlations and tracking of image sharpness and resolution measurements.

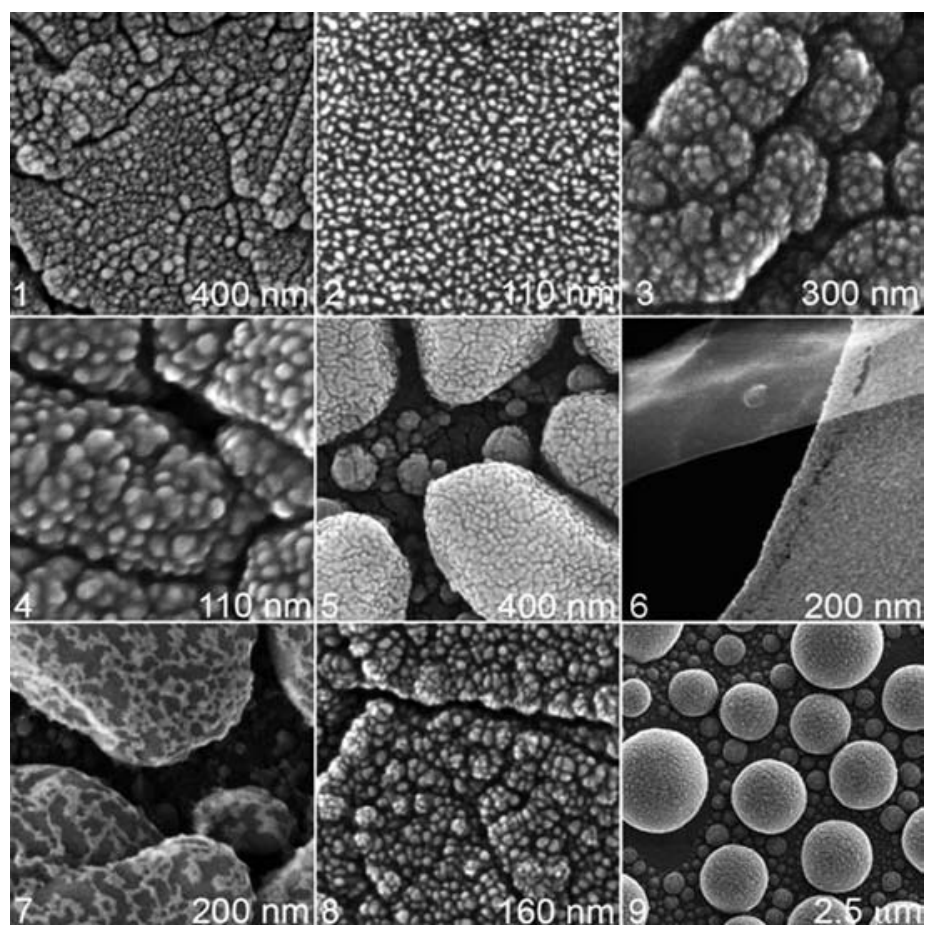

Figure 8. Image series used for the evaluation of image sharpness calculation software candidates. All high-resolution secondary electron images were obtained with the instruments listed in Table 1 and at various landing energies. The field-of-views are shown at the bottom of the images. 


\section{Summary}

For SEMs and HeIMs, there is more to resolution determination than simply laying a ruler on a micrograph and measuring the distance between two points. The resolution of these instruments is a more complex phenomenon than the resolution of the classical light microscope or transmitted electron microscope. Clearly, resolution performance is a very important issue and vast improvements in instrument design have strongly contributed to advances over the past several years. Improved lens designs and illumination sources have been the main contributors to increased instrument performance. Still, the SEM resolution is a function not only of the instrument design, and instrument operating conditions, but also of the material being viewed to demonstrate the performance of the instrument. Particle beam and sample interactions and the signal collection are major factors as well. Hence, sample choice plays a significant role in demonstrating the performance of an instrument. It is also known that the instrument resolution depends upon the materials composition of the sample. For that reason, specialized samples have been developed for the demonstration of resolution capabilities. These samples have had to evolve as did the instruments. For fair evaluations, one must also keep in mind that one sample may work better than another for a specific instrument or a particular set of operating conditions.

\section{Acknowledgements}

The authors would like to thank and acknowledge the effec- tive collaborations with Zeiss SMT, Hitachi High Technologies and FEI Company in the development of this article, and their commitment and continued efforts to improve instrumentation for nanotechnology and nanometrology. The authors would also like to thank both the NIST Office of Microelectronics Programs and International SEMATECH for partially funding this work.

\section{References}

1 Interagency Working Group on Manufacturing R\&D Workshop Report: Instrumentation, Metrology and Standards for Nanomanufacturing. (Available for download www.manufacturing.gov )

2 National Nanotechnology Initiative. NNI Grand Challenge Workshop Report. Instrumentation and Metrology. (available at www.nano.gov).

3 Published specifications for the Hitachi S-5500 SEM [ http://www. hitachi-hta.com/pageloader $\sim$ type $\sim$ product $\sim$ id $\sim 389 \sim$ orgid $\sim 42 . h t m l]$

4 Helium Ion Microscopy. Microscopy and Analysis January 2009 p28 www.smt.zeiss.com/orion

5 Postek, M. T. and Vladar, A. E. 1998. Image Sharpness Measurement in Scanning Electron Microscopy. Part 1. SCANNING 20:1-9.

6 Postek, M. T. and Vladar, A. E. 2008. Helium Ion Microscopy and its Application to Nanotechnology and Nanometrology. SCANNING 30:457-462.

7 Ward, B, Notte, J and Economou, N. 2006. Helium Ion Microscope a New Tool for Nanoscale Microscopy and Metrology. J. VacSci. Technol. B 24(6): 2871-2875

8 Zhang, N-F, Postek, M. T., and R. D. Larrabee. 1999. Image Sharpness Measurement in Scanning Electron Microscopy. Part 3. Kurtosis. SCANNING 21:256-262.

9 Vladar, A. E. and Postek, M. T. and Davidson, M. P. 1998. Image Sharpness Measurement in Scanning Electron Microscopy. Part 2. SCANNING 20:24-34.

$10 \mathrm{http}: / /$ www.iso.org/iso/iso_catalogue/catalogue_tc/catalogue_detail. $\mathrm{htm}$ ? csnumber $=42325$

\section{High Performance Hyperspectral Imaging Solutions for the Life Sciences}

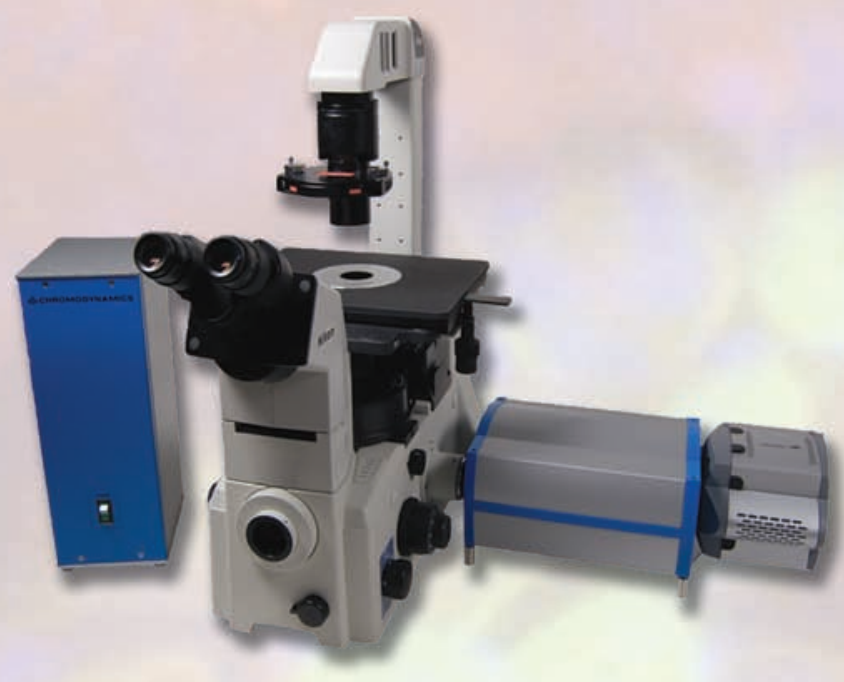

- Immunohistochemistry

- Quantum Dots (Q-dots)

- Fluorescence In-situ Hybridization (FISH)

- Fluorescence Resonance Energy Transfer (FISH)

- Spectral Karyotyping
ChromoDynamics' patented acousto-optic tuneable filter (AOTF) imaging module fulfills the promise of multispectral and hyperspectral imaging through unmatched spectral flexibility and switching speeds.

The AOTF is a high-speed, random access solid state optical filter with an adjustable optical bandpass.

ChromoDynamics' proprietary AOTF technology delivers diffraction limited image quality with variable bandwidths from 1.5 to $25 \mathrm{~nm}$. Wavelength switching time, including computer control overhead, is less than 100 microseconds.

The performance capabilities of this technology make it ideally suited for high-content, high-throughput fluorescence studies, as well as spectral transmission and reflectance imaging. Potential applications range from live cell and whole animal studies to fixed slide clinical diagnostics. 